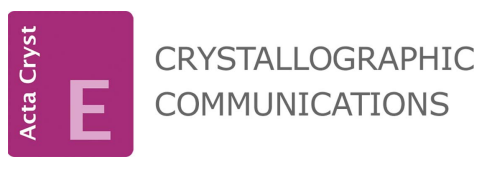

ISSN 2056-9890

Edited by C. Massera, Università di Parma, Italy

Keywords: crystallography; weak interactions; editorial.

\section{Weak interactions in crystals: old concepts, new developments}

\author{
Andrei S. Batsanov* \\ Department of Chemistry, Durham University, Science Site, South Road, Durham DH1 3LE, UK. *Correspondence \\ e-mail: a.s.batsanov@durham.ac.uk
}

\section{Introduction}

In structural chemistry and crystallography, the term 'weak interactions' usually brackets together everything weaker than a single covalent bond or an electrostatic interaction between directly contacting fully charged ions of opposite sign (i.e. an ionic bond). Because it is these forces that hold together a molecular crystal, their study is almost synonymous with the science of organic crystal chemistry - and would require volumes to review. The purpose of the present paper is much more modest: to draw attention to the recent fascinating developments in this field (while also briefly tracing their historical roots) and some unfinished business of the past which now can, and should, be reassessed - and, of course, to provide an introduction to the following research papers.

The theme of this issue is an integrated approach to weak interactions in crystals. The all-too-common pitfall in a crystallographic paper is to make the discussion of geometrical details an end in itself. However, what makes crystal (and molecular) structures stable and drives chemical reactions and phase transitions, is not the geometry per se but free energy, which is not so easy to visualize. Both, in turn, must be stepping stones to understanding, predicting and (hopefully) engineering the properties of crystals. All along, the crystal needs to be seen in a dynamic, rather than static, way - from thermal vibrations to phase transformations to solid-state reactions. ${ }^{1}$

\section{The scope: types of interactions}

Physically, these weak interactions can be classified into Coulombic forces between (usually) not very polar species, the effects of mutual polarization between molecules (polarization forces), the dispersion (van der Waals) forces, and the forces of mutual repulsion between closed electron shells due to the Pauli exclusion principle. The latter, of course, are 'weak' only at or near the equilibrium intermolecular distances and increase exponentially when molecules are forced closer together under pressure, quickly becoming anything but weak. All these forces are ubiquitous in all molecular crystals and beyond, in amorphous solids, liquids and even gases.

Several chemically specific types of weak interactions are often singled out. The hydrogen bond is by far the most important and the most studied - in fact, this concept even predates (Moore \& Winmill, 1912) the discovery of X-ray diffraction. Originally, this term was applied only to $D-\mathrm{H} \cdots A$ interactions where both donor $(D)$ and acceptor $(A)$ were very electronegative atoms $(\mathrm{O}$ and $\mathrm{N}$, but also $\mathrm{F}$ and $\mathrm{Cl})$. The crucial role of such bonds in the structure of water (ice), proteins and DNA is well known. These bonds have energies of $c a 20-40 \mathrm{~kJ} \mathrm{~mol}^{-1}$, while the strongest (charge-assisted or resonance-assisted) hydrogen bonds of $c a 150 \mathrm{~kJ} \mathrm{~mol}^{-1}$ are comparable in energy with covalent bonds proper. Later the concept of the 'hydrogen bond' was expanded, with substantial controversy (Bernstein, 2013), to include 'weak' hydrogen bonds, e.g. C-H $\cdots \mathrm{O}$ ( $c a$ $5 \mathrm{~kJ} \mathrm{~mol}^{-1}$ ) and $\mathrm{C}-\mathrm{H} \cdots \pi$, which can have energies as low as $0.2 \mathrm{~kJ} \mathrm{~mol}^{-1}$, imperceptibly merging into 'unspecified' van der Waals interactions. More recently, Metrangolo and coworkers (Metrangolo \& Resnati, 2001; Metrangolo et al., 2005, 2006) introduced the

\footnotetext{
145 years ago, I heard in a lecture by Professor A. I. Kitaigorodsky, indisputably the founder of organic crystal chemistry, that 'crystal chemistry is to chemistry what autopsy is to surgery'. Great scientists sometimes also make great misjudgements.
} 
concept of the 'halogen bond'. Mono-coordinate $\mathrm{Cl}, \mathrm{Br}$ or I atoms $(X)$ have a depletion of electron density ( $\sigma$-hole) opposite to the covalent bond (Politzer et al., 2007), therefore $Y-X \cdots D$ contacts with electron-donor atoms $D$ can be stabilizing with the energy varying widely, from 10 to $200 \mathrm{~kJ} \mathrm{~mol}^{-1}$. In a similar vein, Scheiner (2013) introduced the pnicogen bond. Meanwhile, so-called ' $\pi-\pi$ interactions' i.e. the causes and effects of parallel stacking of aromatic molecules, remained a disputed issue for a long time, partly due to a mistaken analogy with charge-transfer complexes (see the discussion in Hunter \& Sanders, 1990) and equally fruitless explanations in terms of quadrupole moments (Williams, 1993). Each of these 'bonds' corresponds to a peculiar combination of the forces mentioned above, and should not be regarded as something physically unique; see the illuminating discussion by Dunitz \& Gavezzotti (2005, 2012).

Somewhat aside stands a substantial family of bonds (intraas well as intermolecular) that can be regarded as weakened covalent bonds, or (as sometimes claimed) as 'stills' from the process of making/breaking a chemical bond, tracing a reaction pathway, e.g. of organic addition or elimination, or nucleophilic substitution $\left(\mathrm{S}_{\mathrm{N}} 2\right)$ reactions. After enjoying high popularity in the 1980s and early 1990s (see Bürgi \& Dunitz, 1994), this 'method of molecular correlations' fell out of fashion - but probably deserves reassessment with the present-day tools.

\section{From geometry to energy: four steps forward and a few sideways}

Weak interactions are weak indeed. The total energy of a benzene molecule - calculated by quantum chemistry - is $608 \mathrm{MJ} \mathrm{mol}^{-1}$. Its measured atomization energy, or sum total of covalent bond energy, is $5463 \pm 3 \mathrm{~kJ} \mathrm{~mol}^{-1}$, or less than $1 \%$ of the latter. The sublimation enthalpy, or sum total of intermolecular interactions in a crystal, is a further two orders of magnitude lower, $43-47 \mathrm{~kJ} \mathrm{~mol}^{-1}$ from different measurements. Finally, the energy differences between polymorphs are usually in single units of $\mathrm{kJ} \mathrm{mol}^{-1}$, comparable both to the thermal noise at room temperature $\left(k T=2.5 \mathrm{~kJ} \mathrm{~mol}^{-1}\right)$ and to the error with which the sublimation enthalpy can be measured ( $c a 5 \mathrm{~kJ} \mathrm{~mol}^{-1}$ for organic and $24 \mathrm{~kJ} \mathrm{~mol}^{-1}$ for organometallic compounds, see Acree \& Chickos, 2016, 2017), and beyond the reliability limits $\left(\mathrm{ca} 10 \mathrm{~kJ} \mathrm{~mol}^{-1}\right)$ of the most sophisticated DFT calculations (Mackenzie et al., 2017). This ranking illustrates sharply the intrinsic difficulties of analysing intermolecular forces.

Although the theory of attractive dispersion forces (as interactions of instantaneous dipoles created in atoms by electrons orbiting the nuclei) was first developed by London (1930) who showed that the attractive energy is proportional to $r^{-6}$ where $r$ is the interatomic separation; it was not until 1970 that molecular mechanics calculations of lattice energy became practical. Until that time, molecular crystal structures were interpreted in terms of (i) standard van der Waals radii, compared to actual intermolecular contact distances or used to calculate the packing density (space-filling coefficients), and (ii) hydrogen bonds and other, supposedly specific, interactions between individual atoms. The latter approach probably created the tradition, which persists to the present day, of generally overestimating the significance of such interactions, of the (often misleading) analysis of individual interatomic distances, and of the obsessive search for (progressively weaker) hydrogen bonds. There was, and still is, no reason to depart from the conclusion that 'a significant share of the cohesive potential energy in organic crystals is stored in structurally non-specific molecular contacts that escape a simple taxonomy' (Gavezzotti, 2010). Furthermore, detailed analysis of interactions, on whatever level of sophistication, shows that the shortest (and most conspicuous) intermolecular contacts are not stabilizing at all but repulsive, a 'collateral damage' of the overall optimization of molecular packing (Gavezzotti, 2010).

In the period (roughly) from the 1970s to the beginning of the new millennium, supramolecular structural chemistry was dominated by the so-called atom-atom approximation (Pertsin \& Kitaigorodski, 1987; Filippini \& Gavezzotti, 1993), whereby the lattice energy was represented by a sum of twobody interactions only, the bodies (supposedly) representing atoms in molecules, with all the approximations and arbitrary conventions this entailed. Although the form of these potentials 'descended' from theoretical formulae, such as London's equation, they became essentially empirical formulations, optimized to reproduce correctly macroscopic thermodynamic properties (e.g. sublimation enthalpy) and structural features. On these terms, atom-atom potentials worked remarkably well, but their one-to-one correspondence to physical effects was thus questionable, especially at the microscopic level.

The emergence of the Atoms in Molecules (AIM) theory (Bader, 1990), which successfully rationalized intramolecular electron density in topological terms, created false hopes of intermolecular applications. 'Bond paths' were sought and found in intermolecular space, where electron density was very low and very imprecisely determined in X-ray diffraction experiments. The fashion ended without any lasting benefit, neither in understanding nor in computational utility.

The breakthrough to a physically realistic analysis of crystal packing (made possible, of course, by the immense growth of computer capacity) started around the year 2000 with the development of Hirshfeld surface analysis (Mitchell \& Spackman, 2000; Spackman \& Jayatilaka, 2009), whereby the space occupied by a molecule is defined by partitioning the crystal electron density into molecular fragments. This greatly simplified analysing and visualizing intermolecular contacts, in terms of topological properties of the Hirshfeld surface (shape index, curvedness) and the distances between the surface and nearest atomic nuclei, visualized as 'fingerprint plots' (Spackman \& McKinnon, 2002). The new approach was embodied in the CrystalExplorer software package. At this stage, the analysis allowed is purely geometrical.

The next step was to provide a practical way to (i) calculate intermolecular interactions in a sufficiently precise and physically meaningful way, and (ii) visualize the results in an informative and user-friendly manner. Several such programs 
have been developed (e.g. Johnson et al., 2010). The PIXEL approach developed by Gavezzotti $(2003 a, b, 2010)$ calculates the electron density of a molecule by standard quantumchemical methods, then represents it as a large number of pixel volumes, from which the Coulombic, polarization and dispersion energies can be calculated, using atomic polarizabilities and some other, essentially empirical, approximations and adjustments, to fit experimental sublimation enthalpies. Alternatively, the new CrystalExplorer17 software package (Mackenzie et al., 2017), while using essentially the same (pixel) formalism for the Coulombic term, calculates all other energies by theoretically rigorous quantum-chemical formalisms, with adjustments to a large set of pairwise interaction energies by high-precision quantum methods. For a given pair of molecules, electrostatic, dispersion and total energy of interactions can be calculated and visualized separately. It is no exaggeration that the new techniques are able to revolutionize our understanding of molecular crystallography.

Six of the seven papers of this issue neatly illustrate these different levels of structure interpretation. Yamada et al. (2018) report the structure of a salt with a calixarene anion, a versatile building block of inclusion compounds because of its flexible cavity. Blignaut \& Lemmerer (2018) have studied a series of seven salts composed of primary amine cations and aromatic carboxylate anions. In both works, the dominant supramolecular features are strong 'classical' hydrogen bonds: charge-assisted $\mathrm{N}-\mathrm{H} \cdots \mathrm{O}$ in the latter work, $\mathrm{O}-\mathrm{H} \cdots \mathrm{O}$ in the former; the calixarene-methanol inclusion also results in $\mathrm{O}-$ $\mathrm{H} \cdots \pi$ bonding. Correspondingly, the discussion is focused on the description of these bonds (Blignaut \& Lemmerer using a graphical representation) and other short atom-atom contacts.

Canossa et al. (2018) compare two salts of the methylene blue cation with different anions: $\mathrm{Cl}^{-}$or $\mathrm{HSO}_{4}{ }^{-}$. In both structures the cations are similarly stacked, but in the former they form $\mathrm{N} \cdots \mathrm{H}-\mathrm{O}$ bonds with water of crystallization, but in the latter have none. Surprisingly, this results in drastically different interplanar separations within the stacks: 3.33 versus $3.55 \AA$, respectively, a fine illustration of the need for holistic understanding of a crystal structure. It is tantalizing, however, that the CrystalExplorer energy-calculating facility was not used here, although the Hirshfeld fingerpint plots were generated, but with an older version. Is the rarefied stack really destabilized in terms of energy (as the authors suggest) or is the geometry deceptive? (see above).

The Hirshfeld analysis of a $\mathrm{CoCl}_{2}$ complex with imidazolopyridine (Seth, 2018) captured a remarkable fact, not discussed in the paper: $\mathrm{Cl} \cdots \mathrm{H}$ contacts contribute $30 \%$ (!) of the Hirshfeld surface, although the molecule contains only two chlorine atoms out of 20 potentially accessible nonhydrogen atoms - indicating the importance of electrostatic energy in this structure.

Finally, Geiger et al. (2018) applied the most up-to-date CrystalExplorer17 to analyse the intermolecular energies in the structure of $\mathrm{HOC}_{6} \mathrm{H}_{4} \mathrm{C}_{6} \mathrm{H}_{4} \mathrm{O}\left(\mathrm{CH}_{2}\right)_{9} \mathrm{CO}_{2} \mathrm{Me}$, a long-chain potential gelator, while Dey et al. (2018) do the same for an organocatalyst $\mathrm{PhC}(\mathrm{O}) \mathrm{CF}_{3}$, a liquid under ambient conditions, which they successfully crystallized at $200 \mathrm{~K}$ and characterized at $110 \mathrm{~K}$. It is noteworthy that in the former structure, the largest (!) energy contribution comes from $\mathrm{C}-\mathrm{H} \cdots \pi$ interactions between molecules lying alongside each other, rather than from 'strong' $\mathrm{O}-\mathrm{H} \cdots \mathrm{O}$ hydrogen bonds linking them head-to-tail. Concerning $\mathrm{PhC}(\mathrm{O}) \mathrm{CF}_{3}$, the most striking claim is that the stabilizing energy $\left(-12.7 \mathrm{~kJ} \mathrm{~mol}^{-1}\right)$ of molecular pair III (Fig. 2) can be attributed principally, or even exclusively, to $\mathrm{F} \cdots \mathrm{O}$ and $\mathrm{F} \cdots \mathrm{F}$ attraction. Whereas $\mathrm{Cl}, \mathrm{Br}$ and $\mathrm{I}$ all do form 'halogen bonds' with electron-donor atoms including $\mathrm{O}$ (see Section 2), F is different, having a much smaller $\sigma$-hole. Recently, Sirohiwal et al. (2017) suggested the existence of F...O halogen bonds in two other fluoroorganic compounds, based on exceptionally short F...O contacts in the crystal ( $2.71 \AA$, much shorter than here) and theoretical chargedensity calculations; their conclusions were disputed by Jelsch \& Guillot (2017), whereas an earlier experimental chargedensity study by Pavan et al. (2013) also gave evidence of a $\sigma$-hole on fluorine and donor-acceptor F ...F contacts.

\section{Non-standard conditions}

It is the free energy that is chemically relevant, hence the entropy effects in molecular crystals should not be neglected and at present our knowledge of these is woefully sparse. X-ray structures are seldom studied below $100 \mathrm{~K}$, and for comparison with $a b$ initio calculations the results must be extrapolated to $0 \mathrm{~K}$. Thermal vibrations of molecules in a crystal are dependent on intermolecular forces, as the vibrating particle must 'climb' up the repulsive slope of the potential curve. (It is well-proven that in molecular crystals, intramolecular vibrations are insignificant compared with those of the molecule as a whole.) Badenhoop \& Weinhold (1997) suggested defining the 'natural' van der Waals contact as the distance at which the steric repulsion becomes comparable to the ambient thermal energy $k T\left(2.5 \mathrm{~kJ} \mathrm{~mol}^{-1}\right)$, hence the van der Waals radius must be temperature dependent. This line of research was not developed, but it is obvious that variable-temperature structural studies of molecular crystals can be a substantial help in mapping the intermolecular interactions.

Much more illuminating, however, can be high-pressure studies of such crystals. Indeed, thermal expansion coefficients of organic crystals being of the order of $10^{-4} \mathrm{~K}^{-1}$ (Hofmann, 2002), the volume variation over all practically available temperature ranges can be only a few per cent, whereas the highest compression of a molecular crystal (solid $\mathrm{H}_{2}$ ) achieved so far, reduced its volume 15 times (Batsanov, 2018). Thus, Sikka (2007) analysed the correlations of $\mathrm{O}-\mathrm{H}$ and $\mathrm{H} \cdots \mathrm{O}$ distances in $\mathrm{O}-\mathrm{H} \cdots \mathrm{O}$ hydrogen bonds under pressure, observing the same correlations as had been found at ambient conditions on various compounds - i.e. chemically and pressure-induced deformations are similar. It was also found that the double-well potential of a hydrogen bond is transformed into a single well under pressure. A neutron diffraction study of deuterated $\alpha$-glycine up to $8.7 \mathrm{GPa}$ (Shinozaki et al., 2018) showed non-uniform changes of hydrogen bonds on 
compression, with a large shrinking of bifurcated $\mathrm{N}-\mathrm{D} \cdots \mathrm{O}$ and $\mathrm{C}-\mathrm{D} \cdots \mathrm{O}$ bonds, while strong linear $\mathrm{N}-\mathrm{D} \cdots \mathrm{O}$ bonds change little and in both senses, but in any case, Hirshfeld analysis revealed that the compression was due mainly to squeezing out of voids rather than shrinkage of these bonds. Fanetti et al. (2018) analysed the role of (classical) hydrogen bonds under pressure, in favouring or hindering solid-state reactions (e.g. polymerization of aniline). In this case, shortening $D \cdots A$ distances $d o$ mean stronger bonding, proven by Raman and IR spectra but, surprisingly, instead of lowering the activation energy, it stabilizes the system. This area is poorly understood and requires further research.

High-pressure diffraction experiments using diamond anvil cells (DAC) are still far from routine, notwithstanding the remarkable recent progress in design and availability of such devices (Soignard \& McMillan, 2004). The working volume of a DAC, and hence of the crystal sample, is tiny and the diffraction data correspondingly weak (especially for organic crystals) and is further weakened by the absorption in the much larger diamond crystals, and often overlaps with X-ray scattering from the latter, the gasket, the ruby calibrant and the hydrostatic medium. Metallic parts of a DAC also obscure a large part of the reciprocal space and make it difficult to achieve the necessary completeness of the data. All these factors are essentially unavoidable (although new DACs have wider access angles, see Moggach et al., 2008), but their effects can be greatly reduced by using (i) new X-ray sources with sharper focus and higher intensity, (ii) more sensitive area detectors, especially PILATUS detectors, which do not accumulate noise, as well as (iii) better software, especially for absorption correction. The paper by Zakharov et al. (2018) in this issue, reports a comparative test, whereby the same crystal structure of the thermosalient material 1,2,4,5-tetrabromobenzene was determined using a state-of-the-art instrument or an older-generation diffractometer. The improvement was qualitative!

\section{From energy to properties}

On the whole, this field of research is still in an early stage of development. Probably the most thoroughgoing work by Pulido et al. (2017), is centred on developing 'energy-structure-function' (ESF) maps, i.e. combining computational crystal structure prediction and prediction of properties from the structure, the aim being to engineer highly porous crystal structures (built with intrinsically non-porous molecules) for the purposes of gas storage and guest-molecule selectivity. The lattice energy was calculated using anisotropic atom-atom potentials, and various tools of crystal structure prediction (CSP) were employed, including statistical analysis of known crystal structures. Verifying the ESF map predictions, a new (solvated) form of benzimidazolone was obtained, which was desolvated to yield a material with one-dimensional pores and an extremely low density $\left(0.412 \mathrm{~g} \mathrm{~cm}^{-3}\right)$.

Among the properties most directly related to the anisotropy of intermolecular interactions are the anisotropies of thermal expansion, compressibility and Young's modulus. The calculations of these properties (particularly on various polymorphs of glycine) and their experimental substantiation have been recently surveyed in Mackenzie et al. (2017).

The new capacity to calculate intermolecular interactions reliably and reasonably fast opens up the fascinating prospect of reassessing some long-standing puzzles of structural chemistry. On the other hand, better algorithms for van der Waals forces, thereby developed, may prove useful for understanding and calculating two-dimensional 'vdW-bonded' layered materials, and mixed-dimensional vdW heterostructures (Jariwala et al., 2017).

\section{References}

Acree, W. \& Chickos, J. S. (2016). J. Phys. Chem. Ref. Data, 45, 033101.

Acree, W. \& Chickos, J. S. (2017). J. Phys. Chem. Ref. Data, 46, 013104.

Badenhoop, J. K. \& Weinhold, F. (1997). J. Chem. Phys. 107, 54225432.

Bader, R. F. W. (1990). Atoms in Molecules: A Quantum Theory. Oxford University Press.

Batsanov, S. S. (2018). Shock and Materials. Singapore: Springer Nature.

Bernstein, J. (2013). Cryst. Growth Des. 13, 961-964.

Blignaut, J. \& Lemmerer, A. (2018). Acta Cryst. E74, 580-586.

Bürgi, H.-B. \& Dunitz, J. D. (1994). Editors. Structure Correlations, Vols. 1-2. Weinheim: VCH.

Canossa, S., Predieri, G. \& Graiff, C. (2018). Acta Cryst. E74, 587-593.

Dey, D., Sirohiwal, A. \& Chopra, D. (2018). Acta Cryst. E74, 607-612.

Dunitz, J. D. \& Gavezzotti, A. (2005). Angew. Chem. Int. Ed. 44, 1766-1787.

Dunitz, J. D. \& Gavezzotti, A. (2012). CrystEngComm. 12, 5873-5877.

Fanetti, S., Citroni, M., Dziubek, K., Nobrega, M. M. \& Bini, R. (2018). J. Phys. Condens. Matter, 30, 094001.

Filippini, G. \& Gavezzotti, A. (1993). Acta Cryst. B49, 868-880.

Gavezzotti, A. (2003a). CrystEngComm, 5, 429-438.

Gavezzotti, A. (2003b). CrystEngComm, 5, 439-446.

Gavezzotti, A. (2010). Acta Cryst. B66, 396-406.

Geiger, D. K., Geiger, H. C. \& Morell, D. L. (2018). Acta Cryst. E74, 594-599.

Hofmann, D. W. M. (2002). Acta Cryst. B58, 489-493.

Hunter, C. A. \& Sanders, K. M. (1990). J. Am. Chem. Soc. 112, 55255534.

Jariwala, D., Marks, T. J. \& Hersam, M. (2017). Nat. Mater. 16, 170181.

Jelsch, C. \& Guillot, B. (2017). Acta Cryst. B73, 136-137.

Johnson, E. R., Keinan, S., Mori-Sánchez, P., Contreras-García, J., Cohen, A. J. \& Yang, W. (2010). J. Am. Chem. Soc. 132, 6498-6506.

London, F. (1930). Z. Phys. 63, 245-279.

Mackenzie, C. F., Spackman, P. R., Jayatilaka, D. \& Spackman, M. A. (2017). IUCrJ, 4, 575-587.

Metrangolo, P. \& Resnati, G. (2001). Chem. Eur. J. 7, 2511-2519.

Metrangolo, P., Neukirch, H., Pilati, T. \& Resnati, G. (2005). Acc. Chem. Res. 38, 386-395.

Metrangolo, P., Pilati, T. \& Resnati, G. (2006). CrystEngComm, 8, 946-947.

Mitchell, A. S. \& Spackman, M. A. (2000). J. Comput. Chem. 21, $933-$ 942.

Moggach, S. A., Allan, D. R., Parsons, S. \& Warren, J. E. (2008). J. Appl. Cryst. 41, 249-251.

Moore, T. S. \& Winmill, T. F. (1912). J. Chem. Soc. Trans. 101, 16351676. 
Pavan, M. S., Durga Prasad, K. \& Guru Row, T. N. (2013). Chem. Commun. 49, 7558-7560.

Pertsin, A. J. \& Kitaigorodski, A. I. (1987). The Atom-Atom Potential Method. Berlin: Springer-Verlag.

Politzer, P., Lane, P., Concha, M. C., Ma, Y. \& Murray, J. S. (2007). J. Mol. Model. 13, 305-311.

Pulido, A., Chen, L., Kaczorowski, T., Holden, D., Little, M. A., Chong, S. Y., Slater, B. J., McMahon, D. P., Bonillo, B., Stackhouse, C. J., Stephenson, A., Kane, C. M., Clowes, R., Hasell, T., Cooper, A. I. \& Day, G. M. (2017). Nature, 543, 657-664.

Scheiner, S. (2013). Acc. Chem. Res. 46, 280-288.

Seth, S. K. (2018). Acta Cryst. E74, 600-606.

Shinozaki, A., Komatsu, K., Kagi, H., Fujimoto, C., Machida, S., SanoFurukawa, A. \& Hattori, T. (2018). J. Chem. Phys. 148, 044507.

Sikka, S. K. (2007). High. Press. Res. 27, 313-319.
Sirohiwal, A., Hathwar, V. R., Dey, D., Regunathan, R. \& Chopra, D. (2017). Acta Cryst. B73, 140-152.

Soignard, E. \& McMillan, P. F. (2004). Editors. An Introduction to Diamond Anvil Cells and Loading Techniques. In High-Pressure Crystallography, NATO Science Series II - Mathematics Physics and Chemistry, Vol. 140, edited by A. Katrusiak \& P. F. McMillan, pp. 81-100. Dordrecht: Kluwer.

Spackman, M. A. \& Jayatilaka, D. (2009). CrystEngComm, 11, 19-32. Spackman, M. A. \& McKinnon, J. J. (2002). CrystEngComm, 4, 378392.

Williams, J. H. (1993). Acc. Chem. Res. 26, 593-598.

Yamada, M., Gandhi, M. R., Akimoto, K. \& Hamada, F. (2018). Acta Cryst. E74, 575-579.

Zakharov, B. A., Gal, Z., Cruickshank, D. \& Boldyreva, E. V. (2018). Acta Cryst. E74, 613-619. 